\title{
Criminologie
}

\section{L'étude du processus de changement chez des hommes condamnés à de longues peines}

\section{Dianne Casoni}

Volume 43, numéro 2, automne 2010

Les 50 ans de l’École de criminologie : aperçu de la recherche d'ici et d'ailleurs

URI : https://id.erudit.org/iderudit/1001781ar

DOI : https://doi.org/10.7202/1001781ar

Aller au sommaire du numéro

Éditeur(s)

Les Presses de l’Université de Montréal

ISSN

0316-0041 (imprimé)

1492-1367 (numérique)

Découvrir la revue

Citer cet article

Casoni, D. (2010). L'étude du processus de changement chez des hommes condamnés à de longues peines. Criminologie, 43(2), 351-372.

https://doi.org/10.7202/1001781ar
Résumé de l'article

Le présent article explore la trajectoire de vie de détenus condamnés à des peines à perpétuité mais qui bénéficient d'une libération conditionnelle complète, afin de tenter de mieux comprendre leur processus de changement psychologique. Une méthodologie qualitative a été choisie dans le but d'établir le récit de vie de 20 détenus condamnés à perpétuité mais vivant dans la communauté. Une série d'environ 15 entretiens de deux heures avec chaque participant est prévue pour établir sa trajectoire de vie. À la suite de l'analyse des résultats, les constats préliminaires permettent de dégager deux dimensions importantes dans leurs récits de vie et plus précisément dans leur processus de changement. Ces dimensions ont été désignées par les expressions " moment charnière » et " point tournant ». Le récit de Laurent est présenté pour illustrer ces constats et discuter de leur sens. Enfin, une courte discussion psychanalytique est proposée en conclusion. 


\title{
L'étude du processus de changement chez des hommes condamnés à de longues peines
}

\author{
Dianne Casoni \\ Directrice de la revue Criminologie \\ Professeure titulaire \\ École de Criminologie, Université de Montréal \\ dianne.casoni@umontreal.ca
}

RÉSUMÉ - Le présent article explore la trajectoire de vie de détenus condamnés à des peines à perpétuité mais qui bénéficient d'une libération conditionnelle complète, afin de tenter de mieux comprendre leur processus de changement psychologique. Une méthodologie qualitative a été choisie dans le but d'établir le récit de vie de 20 détenus condamnés à perpétuité mais vivant dans la communauté. Une série d'environ 15 entretiens de deux heures avec chaque participant est prévue pour établir sa trajectoire de vie. À la suite de l'analyse des résultats, les constats préliminaires permettent de dégager deux dimensions importantes dans leurs récits de vie et plus précisément dans leur processus de changement. Ces dimensions ont été désignées par les expressions «moment charnière» et "point tournant». Le récit de Laurent est présenté pour illustrer ces constats et discuter de leur sens. Enfin, une courte discussion psychanalytique est proposée en conclusion.

MOTS-CLÉS - Libération conditionnelle, peine à perpétuité, processus de changement, moments charnière, point tournant, trajectoire de vie, récit de vie.

\section{Introduction}

Alors que le processus de changement chez des hommes condamnés à de longues peines, en particulier ceux condamnés à perpétuité, soulève l'intérêt des chercheurs et des acteurs du système correctionnel, la réaction sociale à ceux-ci donne trop souvent place à l'incrédulité, voire à la méfiance chez nombre de politiciens et de groupes d'intérêt qui voient l'homme condamné à perpétuité comme un être socialement irrécupérable qu'il vaudrait mieux emprisonner à jamais (AFPAD, 
2007). Cet article vise à dépasser de telles représentations sociales qui, malgré leur persistance, cadrent mal avec les constats préliminaires d'une recherche en cours qui permettent de décrire en deux temps le processus de changement observé chez des hommes condamnés à de longues peines, le cas échéant, à perpétuité. Par changement, nous entendons d'abord un changement de perspective psychologique qui conduit ces hommes à modifier leurs attitudes, leurs comportements et leurs styles de vie, dont les comportements criminels font partie. Deux dimensions principales, nommées le «moment charnière» et le «point tournant», ont émergé des analyses préliminaires; elles seront définies, puis décrites à l'aide d'une illustration de cas tirée du récit de vie de Laurent (nom fictif). Puis, en nous inspirant du travail de Debuyst (2010), une courte discussion permettra de relier certains aspects de ces dimensions à des notions psychanalytiques jugées utiles pour mieux comprendre le sens de ce récit.

Toutefois, il serait difficile de traiter de cette question de recherche sans faire aucune référence aux a priori et aux représentations sociales partagés par certains groupes d'intérêt; nous brosserons un tableau sommaire de leurs principaux écrits actuellement en circulation au Québec. Puis, avant de présenter les constats préliminaires de notre recherche, nous tracerons le portrait statistique de la situation des détenus à perpétuité au Canada dans le but de contextualiser notre étude. Ensuite, la méthodologie sera brièvement décrite; les constats préliminaires seront ensuite décrits, puis illustrés à l'aide du récit de vie de Laurent. Une discussion et une conclusion suivront.

\section{«L'incorrigible détenu»}

Le nombre de détenus qui purgent une peine d'emprisonnement à perpétuité est en augmentation constante depuis l'instauration en 1976 de la peine minimale de 25 ans d'incarcération lors de condamnations pour meurtre (National Parole Board, 1999). Toutefois, selon les dernières statistiques disponibles, des 2857 détenus qui purgent une sentence d'emprisonnement à perpétuité au Canada, plus du tiers, soit 1085 détenus, sont en fait en libération conditionnelle et vivent dans la collectivité, soit sous surveillance en maison de transition, soit de façon indépendante, quoique devant se rapporter périodiquement (Comité de la statistique correctionnelle, 2006). Leur libération va à l'encontre du vœu exprimé par certains groupes d'intérêt, comme 
l'Association des familles de personnes assassinées ou disparues (AFPAD) qui plaident, à l'instar de certains politiciens, pour un durcissement des peines pour les auteurs de crimes violents, soutenant qu'il faut protéger la société des auteurs des crimes les plus graves (Harper, 2005; AFPAD, 2007; Boisvenu, 2010; Buzetti, 2010).

Dans un livre populaire qui recense quatre échecs du système correctionnel québécois, Thériault (2005) soutient l'argumentation de l'AFPAD. Il y souligne de plus la faillibilité des programmes de mise en liberté conditionnelle pour certains criminels violents, ce qui l'amène à conclure que l'octroi de permissions de sortie ou de libérations conditionnelles serait un privilège trop facilement concédé.

Il se dégage des écrits de l'AFPAD (2007) et de Thériault (2005), comme d'un certain courant politique actuel au Canada, la représentation, voire la conviction, que malgré le fait que la mission du Service correctionnel du Canada consiste à réhabiliter la population carcérale, les détenus seraient dans l'ensemble des criminels incorrigibles et totalement irrécupérables qu'il vaudrait mieux enfermer à jamais. La conclusion à laquelle ils arrivent est qu'il faut abolir la libération conditionnelle, prolonger les peines et durcir les conditions de détention (Harper, 2005; Thériault, 2005; AFPAD, 2007; Boisvenu, 2010; Buzetti, 2010). Cette conceptualisation du criminel incorrigible est persistante en criminologie. Maruna (2001), Gove (1985) et Farrall et Calverley (2006) constatent que nombre de théories criminologiques, comme celle de l'étiquetage (Becker, 1963) ou de la psychopathie (Hare, 1991), sont basées, implicitement ou explicitement, sur cette notion que le comportement criminel est une conduite qui s'aggrave avec le temps. Selon une telle vision, les conceptualisations de De Greeff (1956) et de Mailloux (1971) de l'homme criminel comme un homme chez qui le comportement criminel peut varier en fonction de facteurs d'ordre émotif, relationnel et social et peut même cesser totalement, seraient tout à fait illusoires.

\section{Les statistiques disent autre chose}

Les données issues de différentes statistiques descriptives de la population carcérale sous surveillance fédérale en libération conditionnelle permettent de tracer un autre portrait des détenus condamnés à des peines à perpétuité. Ces statistiques de récidive permettent de constater que les détenus purgeant une peine à perpétuité mais en libération 
conditionnelle complète, bien que toujours sous surveillance probatoire, récidivent très peu, selon les dernières données disponibles (Instrup, 1992 ; Motiuk et Nafekh, 1997; CNLC, 2005 ; Comité de la statistique correctionnelle, 2006). L'affirmation selon laquelle il s'agirait de criminels incorrigibles et dangereux pour la sécurité publique est donc inexacte. En effet, selon les derniers chiffres disponibles, seulement $0,8 \%$ des hommes condamnés à une peine à perpétuité, mais bénéficiant d'une libération conditionnelle totale, ont été réincarcérés pour un crime en 2004-2005, et aucun d'eux ne l'a été pour un crime violent (CNLC, 2005). Un rapport de la Commission nationale des libérations conditionnelles (CNLC, 2005) ainsi qu'une analyse statistique du Service correctionnel du Canada (Comité de la statistique correctionnelle, 2006) permettent, en outre, de conclure que comparativement à d'autres groupes d'hommes incarcérés, les hommes condamnés à une sentence à perpétuité sont ceux qui récidivent le moins au cours de leur libération conditionnelle.

\section{Le désistement criminel, un processus de changement?}

À ce jour, comme le constatent Farrall et Calverley (2006) ainsi que Maruna et Roy (2007), les connaissances sur le désistement criminel sont éparses et fragmentaires. Peu d'études permettent d'éclairer pleinement le processus qui mène les détenus au désistement. En effet, bien que différentes théories, dont celle de la maturation (Glueck et Glueck, 1940; Gottfredson et Hirschi, 1990), celle du choix rationnel (Cornish et Clark, 1986; Cusson et Pinsonneault, 1986) et celle du contrôle social (Hirschi, 1969; Sampson et Laub, 1993; Laub et Sampson, 2001, 2003), permettent d'isoler certains des éléments ou des motivations qui favoriseraient l'abandon de la criminalité, peu de recherches visent à comprendre comment ces éléments interagiraient ensemble, à travers le temps, et pourraient conduire à un désistement criminel durable.

Depuis l'an 2000, les résultats de quelques études qualitatives ont ouvert une voie dans la compréhension de ce qui est décrit par Warr (2000) et Maruna (2001) comme un processus de désistement criminel. Plusieurs auteurs notent (Warr, 2000; Maruna, 2001; Gadd et Farrall, 2004; Elder et Shanahan, 2006) que le désistement n'est possible qu'à la suite d'une prise de conscience qui conduit l'individu à redéfinir son passé, à revoir sa vie actuelle et à concevoir différemment son avenir, ce qui le conduirait à repenser sa vie en entier. Bien que le processus 
de désistement soit certes plus complexe et comprenne plus de dimensions que la seule dimension psychocriminologique et qu'il dépasse le seul processus qui concerne l'individu incarcéré (Debuyst, 2010; Landreville, 2004), il y a néanmoins lieu d'étudier ce qui se passe pour l'individu en cause, afin d'éclairer cet aspect de la situation.

\section{Méthodologie}

La méthodologie utilisée dans le cadre de cette étude en cours de réalisation est de nature qualitative et d'approche phénoménologique (Churchill, 1990, 2000; Giorgi, 1997). Elle a été choisie dans le but de mieux s'approcher de la réalité subjective des participants (Kvale, 1983; Polkinghorne, 1989). Elle permet, selon Anadon (2006), d'appréhender les significations et le sens que les participants donnent à leurs expériences et à leur vie. Plus précisément, une approche par récit de vie a été choisie (Bertaux, 1986, 1990; Binet et Shérif, 1992; Brochu et Da Agra, 2000) afin de saisir les aspects temporels qui influencent la réalité subjective des acteurs. Cette méthodologie a certes une longue tradition en criminologie. S'inspirant d'une perspective phénoménologique, De Greeff (1927, 1955, 1956) écrivit de belles lignes décrivant ses rencontres avec «l'homme chez le criminel» (1932). C'est à partir d'une méthodologie par récits de vie qu'il dégagea des concepts temporels aussi fondamentaux que le processus criminogène, la dialectique de l'engagement et du désengagement affectif, et l'aliénation progressive associée à la symptomatologie de la haine, pour n'en nommer que quelques-uns. À sa suite, Debuyst (2010) a utilisé la méthodologie du récit de vie couplée à la phénoménologie et parfois à la psychanalyse pour appréhender les situations problématiques qui permettent de jeter un éclairage sur le rôle des acteurs sociaux et de la réaction sociale sur l'étiologie de la délinquance. À l'instar de ces chercheurs, la présente étude utilise la méthodologie du récit de vie afin de cerner ce qui, dans une situation sociale donnée, appartiendrait à un processus de changement psychique et qui aurait comme effet d'éloigner l'individu en cause du processus criminogène dans lequel il était engagé (De Greeff, 1955, 1956). Le processus de changement psychique sera décrit dans les prochaines pages à l'aide de deux dimensions dégagées de l'analyse des récits de vie, soit le «moment charnière» et le "point tournant», puis il sera illustré et enfin discuté à la lumière de quelques notions psychanalytiques. 
À propos de la méthodologie du récit de vie, Farrall (2004) ainsi que Maruna et Immarigeon (2004) sont d'avis que cette approche s'impose lorsqu'il est question d'hommes qui ont connu de longues peines car elle seule permet de saisir les multiples étapes du processus de changement qu'ils traversent. En outre, comme le désistement criminel ne survient pas à la suite d'une décision unique, comme le rappellent De Greeff $(1955,1956)$, Debuyst (2010), ainsi que Farrall et Calverley (2006), mais est le fruit de l'interaction d'un ensemble d'éléments et d'expériences marqués par la temporalité, une collecte de données par récits de vie a été privilégiée. Cette méthode permet non seulement de mettre en relation les expériences passées et présentes des participants (Ahponen, 2005; Bertaux, 2005), mais aussi de cerner les codes, les réactions sociales et les valeurs importantes qui influencent leurs choix et leurs décisions. À ce propos, il est à noter que nombre de criminologues ont utilisé cette méthodologie de recherche dans l'exploration systématique de situations problématiques complexes dont Christian Debuyst en Belgique $(1964,2010)$ ainsi que Serge Brochu, Natacha Brunelle et Marie-Marthe Cousineau au Québec, pour n'en nommer que quelques-uns (Joos et Debuyst, 1968; Brochu et al., 2002; Brunelle et Cousineau, 2005; Debuyst, 2010) ${ }^{1}$.

Les participants à la présente recherche sont tous des détenus, selon la terminologie du Service correctionnel canadien (SCC), puisqu' ils ont été condamnés à des peines à perpétuité. Toutefois, pour être admissibles à la présente recherche, ils doivent bénéficier d'une libération conditionnelle dite totale ou complète, c'est-à-dire qu'ils doivent vivre hors les murs de la prison, librement dans la communauté, bien qu'ils puissent être soumis à des rencontres périodiques avec un agent de libération conditionnelle. Cinq détenus condamnés à perpétuité mais bénéficiant d'une libération conditionnelle complète, sur un total de 20 participants au terme de la recherche, ont déjà été rencontrés entre douze et quinze fois chacun pour des entretiens d'une durée d'une heure trente à deux heures chacun, totalisant environ 30 heures d'entretien.

Les entretiens sont de type semi-directif; les deux premières rencontres visent à appréhender l'histoire du participant de manière générale, alors que les rencontres subséquentes servent à approfondir le récit

1. Suivant une perspective phénoménologique, mais avec un regard sociocriminologique, l'excellent ouvrage d'Anne-Marie Marchietti (2001) donne un aperçu saisissant de la vie des détenus qui purgent de longues peines intra-muros. 
initial. Comme il s'agit d'hommes qui ont à «se raconter» à de nombreuses occasions au cours de leur trajectoire correctionnelle, la première version de leur histoire donne l'impression, souvent, d'être une version superficielle avec laquelle ils sont devenus à l'aise à force de la répéter. Afin d'atteindre un niveau plus grand d'élaboration et d'approfondissement, tout en conservant une attitude semi-directive, le participant est invité au cours des rencontres suivantes à élaborer chacun des aspects narrés au cours des premières rencontres. D'abord, le participant est invité à élaborer sur les évènements marquants, les relations interpersonnelles, les interactions jugées comme significatives et les réflexions qu'il a déjà identifiés dans la première version générale de son histoire comme décisifs ou importants. Le grand nombre d'entretiens (12 à 15) prévus avec chaque participant vise à atteindre un degré élevé d'élaboration et d'approfondissement des thèmes abordés spontanément par celui-ci. Au cours des entretiens d'approfondissement, le participant est aussi encouragé à parler de ses interactions avec les divers acteurs du système judiciaire, des réactions sociales auxquelles il a fait face, mais également de toute autre expérience qu'il juge pertinente.

La confidentialité des informations recueillies est garantie selon les principes d'anonymat proposés par Baez (2002) et Gabbard et Williams (2001). Il s'agit d'omettre tout renseignement nominatif et de déguiser les évènements susceptibles de rendre possible l'identification du participant lors des communications scientifiques subséquentes (Gabbard et Williams, 2001 ; Baez, 2002). Cette procédure est présentée dans le formulaire de consentement, puis décrite et discutée avec chaque participant. Il est à noter que deux participants ont insisté pour que leur récit de vie ne soit pas déguisé, et que leur nom réel soit utilisé. Ils ont expliqué ce choix en disant que leur vie, en raison du crime qu'ils avaient commis, était désormais du domaine public. Malgré ce souhait, par respect pour les familles des victimes, la procédure d'anonymat a été appliquée avec l'accord des deux participants en question.

Les entretiens sont enregistrés (audio) puis retranscrits. L'analyse verticale des entretiens est faite à partir des transcriptions et sert notamment à instruire les chercheurs pour la conduite des entretiens subséquents en repérant les thèmes à approfondir avec chaque participant. Les analyses de discours et de contenu de l'ensemble des entretiens d'un même participant sont soumises à une analyse de consensus. L'analyse transversale de l'ensemble des entretiens ne sera entreprise que lorsque les 20 participants auront été rencontrés et que leurs données d'entre- 
tiens auront été transcrites et analysées, ce qui, compte tenu de l'envergure de la recherche, n'est pas encore achevé. Bien que préliminaires, les analyses effectuées à ce jour possèdent les caractéristiques essentielles d'une méthodologie qualitative rigoureuse et d'une analyse par trajectoire de vie (Bell, 2005), c'est-à-dire qu'elles ont été faites selon un mode inductif, sont rigoureuses et ancrées dans les données empiriques recueillies (Poupart et al.,1997; Bertaux, 2005). Les constats préliminaires d'analyse qui suivent seront néanmoins mis à l'épreuve par les suites de la recherche.

\section{Constats préliminaires d'analyse}

Lanalyse des entretiens des cinq premiers participants a permis de dégager deux dimensions principales. Ces deux dimensions sont désignées par les expressions «moment charnière» et "point tournant», qui renvoient à deux temps distincts dans le cheminement des hommes rencontrés. La première expression, "moment charnière», est utilisée pour décrire un événement, habituellement de nature interpersonnelle, où quelque chose de significatif se passe dans le rapport à l'autre. Il semble, en effet, que certaines interactions, lorsqu'elles se produisent en un moment opportun, ont le potentiel de favoriser une remise en question chez l'individu. Les moments charnières marquent ainsi le temps - au cours de ce long processus de changement psychique - et désignent les moments où un évènement, surtout de nature interpersonnelle, provoque soit une remise en question de sa vie présente, une réflexion sur son passé ou encore une interrogation fructueuse sur son potentiel de changement à venir. Quant au "point tournant», cette expression décrit ce qui est vu par les participants, rétrospectivement, comme ayant constitué un moment charnière particulièrement porteur de sens (Debuyst, 2010), au point où la trajectoire même du participant en est infléchie.

Même si le moment charnière et le point tournant peuvent être représentés par la même interaction ou le même événement relationnel, il s'agit néanmoins de deux dimensions distinctes de la trajectoire en ce qu'elles correspondent à deux temps distincts dans l'histoire subjective que l'individu reconstitue de sa vie. De plus, alors qu'il peut y avoir plusieurs moments charnière qui marquent la trajectoire de l'individu et qui, à son avis, l'ont forcé à réfléchir sur lui-même, ou encore ont favorisé une remise en question à des moments opportuns dans sa vie, 
chaque moment charnière ne constituera pas forcément, a posteriori, ce que le participant considérera comme un point tournant dans sa trajectoire. Le moment charnière décrit ainsi un événement, surtout de nature relationnelle, qui a favorisé la réflexion chez l'individu sur le sens de sa vie et, bien qu'il survienne à un moment opportun de sa trajectoire, ne constitue pas nécessairement un point tournant dans sa vie. Le point tournant, en ce sens, décrit un infléchissement de la trajectoire que parfois seul le participant est en mesure d'identifier lorsqu'il considère sa vie rétrospectivement. Mais le plus souvent, le point tournant est manifeste dans la trajectoire des participants. Il s'agit d'un événement relationnel ou d'une interaction qui a pris un sens particulier pour l'individu et marqué de manière évidente le reste de sa trajectoire.

Le récit de vie de Laurent sert à illustrer le processus de changement psychique observé chez les participants à notre étude. Les deux dimensions qui caractérisent ce processus de changement, soit le moment charnière et le point tournant, sont également illustrées par ce récit qui sera discuté plus loin à l'aide de quelques notions psychanalytiques.

\section{Laurent}

Les entretiens avec Laurent ont certainement contribué à soutenir l'idée que l'évènement relationnel constitue l'étape nécessaire à la constitution d'un moment charnière dans la trajectoire d'un détenu condamné à perpétuité mais qui bénéficie d'une libération conditionnelle dite complète. Il serait impossible, en utilisant uniquement des extraits d'entretien, de rendre compte d'une aussi longue trajectoire de vie que celle de Laurent - plus d'une cinquantaine d'années -, ainsi, nous recourons à la fois à des extraits d'entretiens et à des résumés de sa trajectoire afin de respecter tant les exigences méthodologiques que les contraintes d'espace.

Laurent est l'aîné de quatre enfants, nés à une année d'intervalle chacun. Au cours de leur enfance, il arrivait fréquemment que leur père, qui était selon Laurent un joueur alcoolique et violent, réquisitionne le logement où ils vivaient pour y tenir des parties de cartes illégales qui duraient habituellement plusieurs jours. Selon le récit de Laurent:

Il [père] ouvrait la lumière dans notre chambre et nous criait: «Dehors!» en nous donnant des coups de poing, puis des coups de pied partout. On [n']avait pas le temps de s'habiller ni rien, même pas notre manteau, l'hiver. 
Même ma mère, tu sais, il la jetait dehors à coups de pied en jaquette sans une cenne pour nous faire manger. Je l'haïssais!»

Laurent raconte qu'il a été confronté très jeune au besoin de se «débrouiller pour survivre». Il précise qu'il devait, avec ses frères, sa sœur et sa mère: «[se] débrouiller pour trouver à manger, pour trouver une place pour coucher, se trouver du linge, pour pas geler l'hiver». En tant qu'aîné, il dit qu'il se sentait responsable de ses frères et sœur, mais aussi de sa mère dont il dit: «c'était une enfant, comme nous». Lorsqu'il a été invité à élaborer cette affirmation, il en est venu à décrire sa mère comme étant incapable de prendre des décisions, de protéger ses enfants ou de se protéger elle-même des comportements violents et des abus de son mari. Il donne comme exemple le fait que c'était leur tâche commune, à son frère cadet et à lui-même, de trouver des cachettes pour se réfugier le jour dans la ville, là où il y avait des bouches d'air chaud qui émanait des édifices. En parlant de sa mère, il dira: «c'est qu'elle a été trop battue par son mari, puis ça l'a rendue comme zombie. Elle, elle avait perdu son instinct de survie. Une chance qu'on était là des fois parce que sinon elle se serait laissée mourir, je pense.»

Laurent raconte avoir peu fréquenté l'école en raison du comportement de son père et du fait qu'ils étaient souvent forcés d'errer sans accès à leur logement. Il ajoute que, lorsqu'il y allait, il ne s'y sentait pas à sa place. Il raconte avoir eu l'impression que tous les yeux étaient sur lui et qu'on le surveillait de près. Lorsqu'il a été invité à élaborer sur ses impressions et ses expériences scolaires, Laurent répond qu'il se battait fréquemment dans la cour de récréation parce qu'il ne savait pas parler. Interrogé sur ce qu'il voulait dire par là, il précise qu'il n'avait pas une bonne élocution, mais surtout qu'il ne savait que "parler avec [s]es poings». En raison de son comportement batailleur, Laurent dit qu'il se retrouvait souvent au bureau du directeur, qu'il haïssait intensément. Il réalise en parlant de cet homme au cours des entretiens qu'il le haïssait sans raison véritable car, dit-il:

Le principal... dans le fond... il a toujours été correct avec moi. [...] Il a vraiment essayé de m'aider, il me parlait, il [ne] m’a pas donné la strap même si je faisais semblant que c'est ce qui se passait dans son bureau... il faisait juste me parler ... pas fort, à part ça, il [ne] criait pas, rien... mais, je [ne] lui faisais pas confiance... Je [ne] faisais confiance à personne, tous les hommes étaient comme mon père pour moi dans ce temps-là! 
Laurent raconte que, pendant longtemps, il voyait son père dans tous les hommes qu'il rencontrait et qu'il les haïssait tous, "qu'ils soient corrects ou pas [avec moi], je [n']étais pas capable de les voir en peinture, j'étais juste trop enragé contre mon père». La rage que décrit Laurent à l'égard de son père semble avoir eu une qualité obsessionnelle en ce qu'elle l'a accompagné dans tous les instants de sa vie des années durant. En plus de se battre très souvent avec des pairs, Laurent s'adonnait au vol de biens de toute nature au cours de son adolescence. À 17 ans, il rapporte avoir été arrêté par vol et, trouvé coupable, il a reçu une sentence d'emprisonnement à servir dans une prison pour adultes, où il rapporte avoir été une proie facile pour des détenus plus âgés. Il élabore peu sur les épisodes de viols subis à son arrivée en prison, il y fait référence toutefois pour justifier son besoin de renforcer sa musculature et de prendre du poids afin de ne plus être un objet de convoitise ou du moins pour pouvoir s'en protéger s'il l'était. Laurent raconte qu'il a passé en conséquence cette année d'incarcération: «à faire des poids et haltères» tout en nourrissant des projets de vengeance contre celui qui, croyait-il, l'avait dénoncé pour le vol pour lequel il purgeait une peine d'incarcération.

Dès sa sortie de prison, Laurent rapporte qu'il a cherché à s'acheter une arme et qu'il a pris la première qu'on lui a proposée: «... je [ne] pensais pas aux conséquences, j'avais juste une idée en tête, c'était de le faire payer sa trahison", dit-il en parlant de celui qui, croyait-il, l'avait dénoncé. Il l'a trouvé, raconte-il:

[...] à la salle de pool, où on avait l'habitude de se tenir. Je m'en souviens comme si c'était hier, il était accoté sur une table [de billard] puis il riait. Quand il m'a vu, il a arrêté net de rire et il a commencé à essayer de s'expliquer, mais il n'a pas eu le temps de rien dire parce que je l'ai tiré, beau cave, devant tout le monde qui était là. Tu sais, c'était plus important pour moi de montrer à tout le monde que je [n']étais pas quelqu'un qu'on pouvait trahir sans payer pour, que d'avoir peur de me faire prendre. J'étais malade de même.

Laurent raconte que moins de 48 heures après sa sortie de prison, il a été de nouveau incarcéré et condamné à une peine d'emprisonnement à perpétuité pour meurtre au premier degré, soit meurtre prémédité. Il dit qu'une des répercussions de son geste a été de consolider la réputation de dur à cuire qu'il voulait avoir. Il raconte que ce second séjour s'est déroulé mieux pour lui car il était craint des autres détenus, mais 
aussi, dans une certaine mesure, des gardiens. Il explique qu' il était non seulement très fort physiquement, mais aussi très impulsif: «[...] tu sais, parce que j'avais la réputation d'être un tueur de sang froid (sic), ils [gardiens et pairs] avaient peur de mes réactions». Laurent raconte qu'il jouissait d'un certain prestige et que cela le réconfortait d'une certaine manière, même s'il trouvait la vie pénitentiaire dure, car il faisait souvent l'objet de mesures disciplinaires et était soumis à l'isolement. Laurent précise que son statut, il le devait aussi au fait qu'il prenait toujours partie pour les détenus et que cela entraînait souvent des mesures disciplinaires. Il raconte qu'il n'avait malgré cela qu'une idée en tête, celle de s'évader, et que ses très nombreuses tentatives d'évasion ratées, seul ou avec d'autres, lui ont valu de très fréquents et longs séjours en cellule d'isolement.

Malgré ces échecs, il n'avait que l'évasion en tête et raconte avoir réussi à s'évader plusieurs fois pour des périodes de temps variables. Une évasion en particulier a été longue, rapporte Laurent qui, cette fois-là, non seulement s'est évadé mais a aussi volé une somme très importante. Il raconte qu'il est resté en cavale pour une dizaine d'années et que ça n'a été que sur dénonciation de son complice pour le vol qu'il a été retrouvé et condamné pour l'évasion et le vol. Laurent raconte qu'au moment de sa reprise:

Je vivais à $\mathrm{X}$ avec Ginette [nom fictif] depuis deux ans à peu près. C'était la première fois de ma vie que j'étais en amour. On vivait une vie simple, on avait une maison comme les autres, rien de trop fancy, rien pour nous faire remarquer. J'étais heureux avec elle. Je n'ai pas eu le temps de m'enfuir quand le gars avec lequel j'ai fait le vol s'est fait prendre... je l'ai su trop tard. C'était comme dans un film, tu sais, quand la police m'a repris. J[e n]'ai pas résisté, je [ne] voulais pas qu'ils tirent, qu'il y ait de coups de feu parce que je [ne] voulais pas que Ginette se fasse tirer. J[e n]'avais jamais rien dit à Ginette du vol ou d'où était caché l'argent parce que je [ne] voulais pas qu'elle soit complice si je me faisais prendre. Elle [ne] savait rien. Quand j'ai vu la police se placer autour de la maison, je savais que j'étais pris puis je lui ai dit de [ne] pas m'attendre, de refaire sa vie... que je m'en allais en-dedans pour longtemps. C'est plus tard, une fois en dedans qu'elle m'a dit qu'elle était enceinte de moi.

Bien que Ginette ait accepté de «refaire sa vie» rapporte Laurent, elle le visitait régulièrement au pénitencier avec leur enfant, Jean-Yves (nom fictif). Quant à Laurent, il raconte qu'il jouissait d'un grand prestige à son retour au pénitencier en raison de la durée de son évasion et de la 
somme importante qu'il avait volée. Il précise que Ginette continuait de venir le visiter avec Jean-Yves. Lors de la scène suivante que Laurence décrit, Jean-Yves avait environ huit ans:

Ils arrivaient pour une visite de roulotte et puis moi, beau niaiseux, j'avais encore essayé de m'évader et puis là il y avait deux gardiens qui m'escortaient au trou et puis on s'est trouvé à passer vis-à-vis l'entrée des visiteurs, mais de l'autre côté de la vitre, du côté du pénitencier en même temps que, de l'autre bord, il y avait Ginette et puis Jean-Yves qui arrivaient pour la roulotte de la fin de semaine. Puis là, Jean-Yves, il me voit, les mains attachées à la ceinture, puis les pieds avec des grosses chaînes, tu fais dur devant ton fils, t[u n']as pas l'air fin, fin. À travers la grosse vitre épaisse, j'entends Jean-Yves me crier: "Quand est-ce que tu vas arrêter de faire le con? On [ne] va jamais vivre ensemble si tu continues de même!» Moi, j'ai rien dit. Je suis resté l'air bête, mais j[e n]'ai pas arrêté d'y penser tout le temps que j'ai été dans le trou, puis j'ai été là longtemps!

Laurent rapporte que la charge affective avec laquelle Jean-Yves s'est exprimé a résonné en lui au cours de son long séjour en isolement. Il ajoute que l'idée même de vivre avec son fils, à laquelle il n'avait jamais osé penser, a commencé à ce moment-là à se poser en lui comme une possibilité. Il explique que, jusqu'à ce moment-là, les projets d'évasion, de vols ou de vengeance occupaient l'essentiel de sa pensée. Le cri de Jean-Yves à son endroit est venu bouleverser, raconte Laurent, sa façon de penser, ses plans d'avenir et, à terme, jusqu'à sa façon d'être avec lui-même et avec les autres. Comme exemple de sa façon habituelle de penser jusqu'alors, Laurent raconte qu'au moment où Jean-Yves s'est adressé ainsi à lui, de son côté il avait le projet de s'enfuir, à la première occasion, à jamais, loin du Québec, coupant donc tout contact avec ceux qu'il connaissait, y compris Ginette et Jean-Yves, avec l'idée de se refaire une nouvelle identité. Il raconte qu'étant en isolement, il n'a pas pu faire autrement que de penser et repenser aux paroles de Jean-Yves et que leur charge émotive et leur signification ont fini par le confronter dans sa façon de penser. L'idée de voir Jean-Yves grandir, de jouer un rôle dans sa vie et même d'avoir une certaine responsabilité par rapport à lui et vis-à-vis Ginette s'est présenté à lui peu à peu, explique-t-il.

Laurent raconte être resté plus d'un mois en isolement à la suite de cette tentative d'évasion. Il précise, en outre, que c'est au cours de ce séjour en isolement que sa décision de changer de vie a pris forme à la suite des réflexions que le cri de cœur de Jean-Yves a suscitées en lui. Il en parle ainsi: 
Je me suis rendu compte que je lui devais ça [à Jean-Yves] et que je devais faire tout ce que je pouvais pour avoir une libération conditionnelle. Je savais que ce n'était pas gagné, je me disais que la Commission [de libération conditionnelle] se méfierait de moi et je comprenais ça, j'aurais fait pareil à leur place... après ce que j'avais fait. Je me disais que ça se pouvait que je n'aie jamais de libération conditionnelle [...]. Ça se pouvait que je ne réussisse jamais à obtenir une libération conditionnelle, tu sais, mais il fallait que je fasse tout ce que je pouvais, au moins pour que Jean-Yves sache que j'avais essayé.

Bien que Laurent raconte que tout a basculé en lui au cours de ce séjour en isolement, il demeure plutôt avare de détails sur le contenu précis de ses pensées. Il a tendance à résumer les grands thèmes de remises en question et de prises de conscience qui ont eu cours en lui pendant cette période de sa vie. Certaines grandes idées générales comme celle d'avoir repensé à l'ensemble de sa vie, à ses expériences en amitié, à son sentiment d'avoir été répétitivement trompé par ceux en qui il avait le plus confiance, à sa crainte que l'avenir soit une répétition du passé, avec ses trahisons, ses impasses et son sentiment de solitude. Laurent raconte comment, progressivement, le désir qu'exprimait son fils de vivre avec lui est aussi devenu le sien. En parlant des diverses étapes qu'il a traversées, il observe que ce n'est qu'en les traversant qu'il s'est rendu compte des défis auxquels chacune le confrontait. Il donne l'exemple d'avoir dû se confronter à la nécessité de changer complètement de fréquentations et même d'amis dans le pénitencier afin de gagner la confiance des gardiens, mais aussi afin de montrer à ses pairs qu'il ne voulait plus être mêlé aux situations de la vie courante qui l'auraient amené à commettre des actes délinquants. Il précise que ce changement a été le plus difficile car ses amis le voyaient comme un traître et les gardiens croyaient à une ruse: «Ça[n'a] pas été facile, ni les gardiens ni les gars [n']avaient confiance en moi. Ils me voyaient comme un stool ou bien comme un menteur. Il [n'y] avait personne qui me croyait.» Il dit qu'au cours de cette période, il s'est senti très seul, mais en même temps: «je ne pouvais plus retourner en arrière, en dedans de moi, c'était clair, c'était décidé, je ne voulais plus tromper personne, plus de vols, plus de violence, je ne voulais plus intimider personne».

Cette période semble bien correspondre à un moment charnière dans le processus de changement de Laurent qui commence à découvrir, selon ses termes, «de nouvelles valeurs» morales pour guider ses actions. La 
décision de changer de fréquentations et d'amis a été sans doute une des plus importantes de son processus de changement puisqu'elle lui a demandé beaucoup de courage; entre autres parce que cette décision ne pouvait pas passer inaperçue et qu'elle a entraîné des conséquences importantes dans sa vie quotidienne. Les effets de cette décision sur ses relations avec ses pairs et sur son statut auprès d'eux ont été multiples et se sont déclinés différemment au fur et à mesure que les années ont passé et que sa loyauté a été mise à l'épreuve. Puis un événement dramatique est venu lui donner un nouveau rôle:

Puis, là, il y a eu l'émeute, c'est là que ça a commencé à changer. Les gars ont pu voir que je [n'] ai pas stoolé personne, puis que je suis resté avec eux autres, mais, en même temps, les gardiens ont vu que je n'ai pas participé à l'émeute, mais [que] je les ai aidés en parlant aux gars et puis en les calmant pas mal quand ça a commencé à chauffer trop.

Laurent semble rendre compte dans cet extrait du moment où le changement dans ses rapports avec ses pairs et avec les gardiens, bien que mis en place des années plus tôt, a conduit à une issue positive. Cette issue, favorable dans ses rapports avec les autres, semble constituer un autre moment charnière dans sa trajectoire.

Le processus de changement enclenché chez Laurent depuis les paroles de son fils lors de sa tentative d'évasion ratée jusqu'à sa libération conditionnelle en maison de transition a duré plus de quinze ans. Laurent désigne, en rétrospective, le moment charnière constitué par les mots de son fils dans un moment de grande vulnérabilité narcissique chez lui comme un point tournant à partir duquel tout a changé dans sa vie. Pour notre part, nous suggérons que son investissement relationnel a effectivement changé de direction, allant d'un investissement centré exclusivement sur soi-même vers un investissement des personnes composant son champ relationnel, d'abord constitué de son fils, la mère de celui-ci, ses frères, sa sœur et sa mère, puis d'un champ relationnel élargi composé de ceux dans le milieu pénitencier, puis dans la communauté élargie. Ce changement de direction de ses investissements relationnels a conduit Laurent à effectuer d'importants renoncements. Par exemple, en choisissant d'investir Jean-Yves, il a dû renoncer à exercer sa vengeance contre son propre père, et par voie de conséquence, contre toutes les figures d'autorité qui pouvaient représenter ce dernier symboliquement. Laurent observe, à ce propos, qu'en cessant de haïr son père, il a constaté que ses rapports sociaux se sont transformés: 
Tu sais, être dans le métro, maintenant... être juste «comme les autres», ça m'apporte plus que quand j'étais le boss. Ça l'air drôle à dire, mais je n'ai plus besoin de ça, de faire peur aux autres. Je me fais bousculer dans le métro, puis le sourire me vient aux lèvres presque. Avant j'aurais sorti les gros bras si quelqu'un m'avait fait moins que ça. Puis, c'est drôle, je suis plus heureux d'être comme maintenant, d'être comme tout le monde, qu'avant d'être le plus fort puis de faire peur à tout le monde.

\section{Discussion}

Différentes trajectoires ont été identifiées chez les hommes interviewés; nous observons toutefois, comme le suggèrent Bushsway et ses collaborateurs (2001), une séquence temporelle propre au processus de changement au sein de chacune de celles-ci. Le cas de Laurent montre bien cette séquence temporelle: une rencontre inattendue avec son fils à un moment où il était dans une situation de défaite, voire de vulnérabilité narcissique, a donné lieu à un moment charnière dans sa trajectoire de vie, moment charnière qui est vécu, après coup, comme un véritable point tournant lorsqu'il raconte sa vie. Il y a lieu d'observer que Laurent avait sûrement déjà connu des moments de vulnérabilité narcissique dans le passé; toutefois, cette fois-là, il a été surpris, en un moment de plus grande sensibilité. Ce n'est qu'une fois que la vision clivée du monde, en termes de lutte de pouvoir entre forts et faibles, agresseurs et victimes, a été ébranlée en lui que les premiers changements d'attitudes et de comportements ont commencé à s'exprimer (Casoni et Brunet, 2002, 2007). L'abandon progressif d'une telle vision clivée du monde se dégage avec netteté de l'analyse du discours de Laurent. À partir du moment où les gardiens n'étaient plus vus d'emblée comme des agresseurs, le recours à l'identification à l'agresseur n'a plus été nécessaire, non plus, pour se protéger d'eux, d'où la capacité de Laurent d'adopter un comportement neutre de pacificateur et de négociateur lors de l'émeute. Un autre exemple serait quand Laurent en est arrivé à cesser d'en vouloir à son père au point de vouloir le tuer. Ceci a libéré du coup sa psyché des pensées compulsives de vengeance qui l'occupaient et a diminué le besoin de recourir à l'identification à l'agresseur pour se protéger de la menace que son père vengeur, à son tour, représentait pour lui - de même que de la menace de toutes les figures d'autorité qui symbolisaient le père. 
Laurent exprime les effets de ces modifications de son équilibre psychique lorsqu'il relate comment il a été étonné de constater que lorsqu'il s'est mis à parler de façon respectueuse aux autres, et surtout aux gardiens, ceux-ci ont été respectueux à leur tour: "J'ai découvert que je n'étais pas toujours obligé de sortir les gros bras dans la vie pour me faire respecter, pour ne pas me faire écraser par les autres.» C'est ainsi que le processus d'identification empathique aux autres a été activement soutenu par l'abandon momentané, puis progressif, du complexe défensif assez caractéristique de l'équilibre psychique du délinquant violent, soit le déni, le clivage et l'identification à l'agresseur. Lorsque Laurent dit que ce qui a fondamentalement changé en lui ce sont ses valeurs, il n'a certes pas tort. Il y a lieu toutefois d'apprécier que ce changement de valeurs repose davantage sur une représentation nouvelle de lui-même et d'autrui plutôt que sur une simple décision de changer de valeurs. Dès que son regard sur l'autre n'a plus été déformé par l'identification à l'agresseur, il a pu s'identifier à autrui plus facilement, ce qui a soutenu sa capacité naissante d'empathie. Ses valeurs sont certainement nouvelles, mais ce qui lui a permis d'adhérer à de nouvelles valeurs, c'est le fait d'être désormais capable d'accueillir en lui-même une représentation non menaçante d'autrui.

\section{Conclusion}

Les résultats de recherche présentés visaient à identifier les composantes du processus de changement qui a cours chez des détenus dont le processus de réhabilitation présente de grands défis en raison de la gravité de leurs actes criminels. Comme les statistiques le démontrent bien (CNLC, 2005), leur réinsertion sociale est possible et même particulièrement réussie. Cependant, ce que les résultats préliminaires de cette recherche ont permis de constater est que ce processus de changement est enclenché par la rencontre avec l'autre à un moment de vulnérabilité psychique chez le détenu. Dans ces occasions opportunes, de telles rencontres donnent lieu à de véritables moments charnière dans la trajectoire de ces hommes qui, rétrospectivement, se les représentent comme un point tournant dans l'histoire de leur vie, point tournant qui les conduit à une redéfinition d'eux-mêmes et des autres.

Bien que l'on puisse supposer qu'un processus de changement qui repose sur une telle redéfinition de soi doive passer par un processus d'introspection de type psychothérapique, il est des individus qui, 
comme Laurent, ont mené seuls un processus réflexif d'introspection. Toutefois, la grande majorité des détenus rencontrés estiment qu'il leur a été nécessaire de suivre une psychothérapie et jugent cet apport essentiel dans leur processus de changement. À ce sujet, il appert qu'un des défis importants dans le cheminement des hommes condamnés à de longues peines, a fortiori à des peines à perpétuité, consiste à se pardonner suffisamment leurs crimes pour se donner moralement le droit de vivre. En ce sens, il serait intéressant, dans la seconde étape de cette recherche, de tenter de mieux comprendre les enjeux et les vicissitudes du rapport que ces hommes entretiennent avec leurs sentiments de culpabilité et de faute.

\section{Références}

AFPAD (2007). Communiqué de presse. Association des familles de personnes assassinées ou disparues (AFPAD). Disponible sur: www.afpad.ca/fr/ communiques/20_04_2007.shtml

Ahponen, P. (2005). Precariousness of everyday heroism. A biographical approach to life politics. Qualitative Sociology Review, I (2), 22-42.

Anadon, M. (2006). La recherche dite «qualitative»: de la dynamique de son évolution aux acquis indéniables et aux questionnements présents. Recherches qualitatives, 26 (1), 5-31. Disponible sur: www.recherche-qualitative.qc.ca/ Revue.html

Baez, B. (2002). Confidentiality in qualitative research: reflections on secrets, power and agency. Qualitative research, 2 (1), 35-58. Disponible sur: http:// qri.sagepub.com/cgi/content/abstract/2/1/35

Becker, H. (1963). Outsiders. Études de sociologie de la déviance. Paris: Metailié.

Bell, A. J. (2005) Oh yes, I remember it well! Reflections on using the life-grid in qualitative interviews with couples. Qualitative Sociology Review, I (1), 51-67.

Bertaux, D. (1986). Fonctions diverses des récits de vie dans le processus de recherche. In D. Desmarais \& P. Grell (Eds.), Les récits de vie. Théorie, méthodes et trajectoires-types (21-34). Montréal: Éditions Saint-Martin.

Bertaux, D. (2005). L'enquête et ses méthodes. Les récits de vie. Paris: Armand Collin.

Binet, L., \& Shérif, T. (1992). Les récits de vie: mode d'emploi. Revue canadienne de service social, 9 (2), 183-200.

Boisvenu, P.-H. (2010). Criminalité: le sénateur Boisvenu parle de manipulation. Cyberpresse. 21 juillet 2010, mis à jour le 21 juillet 2010, www. cyberpresse.ca/actualites/quebec-canada/politiquecanadienne/ 201007/21/01-4299959-criminalite-le-senateur-boisvenu-parle-demanipulation.php ? utm_categorieinterne $=$ trafficdrivers $\&$ utm contenuinterne $=$ envoyer_cbp 
Brochu, S., Da Agra, C., \& Cousineau, M.-M. (Eds.) (2002). Drugs and Crime: Deviant Pathways. Leichester: Ashgate Publications.

Brunelle, N., \& Cousineau, M.-M. (Eds.) (2005). Trajectoires de déviance juvénile: les éclairages de la recherche qualitative. Ste-Foy: Les Presses de l'Université du Québec.

Bushway, S. D., Piquero, A. R., Broidy, L. M., Cauffman, E., \& Mazerolle, P. (2001). An empirical framework for studying desistance as a process. Criminology, 39, 491-515.

Buzetti, H. (2010). Le crime ne paie pas, mais les programmes coûteront cher. Le Devoir.com, 29 avril 2010. Disponible sur: www.ledevoir.com/politique/ canada/287944/le-crime-ne-paie-pas-mais-les-programmes-couteront-cher

Casoni, D., \& Brunet, L. (1998). Violence et impasses en psychothérapie: de l'utilisation de l'objet par la fonction contenante. In F. Millaud (Ed.), Actes de violence: réflexions psychodynamiques et cliniques (chapitre 8). Paris: Masson (édition de 2009).

Casoni, D., \& Brunet, L. (2002). La psychocriminologie. Apports psychanalytiques et applications cliniques. Montréal: Presses de l'Université de Montréal.

Casoni, D., \& Brunet, L. (2007). The psychodynamics that lead to violence. Part 1; The case of the chronically violent delinquents. Canadian Journal of Psychoanalysis, 15 (1), 41-55.

Churchill, S. D. (1990). Considerations for teaching a phenomenological approach to research. Journal of Phenomenological Psychology, 21 (1), 46-47.

Churchill, S. D. (2000). 'Seeing through" self-deception in narrative reports: Finding psychological truth in problematic data. Journal of Phenomenological Psychology, 31 (1), 44-62.

Churchill, S. D. (2006). Phenomenological analysis: Impression, formation during a clinical assessment interview. In C. T. Fisher (Ed.), Qualitative research methods for psychologists: Introduction through empirical studies (79-110). San Diego, CA: Elsevier.

Comité de la statistique correctionnelle (2006). Aperçu statistique: Le système correctionnel et la mise en liberté sous condition, 2006. Comité de la statistique correctionnelle. Travaux publics et Services gouvernementaux Canada. No de cat.: PS1-3/2006F.

Commission nationale des libérations conditionnelles (2005). Rapport annuel sur le rendement de la Commission nationale des libérations conditionnelles. Rapport présenté au ministre de la Sécurité publique et de la Protection civile. Disponible sur: www.npb-cnlc.gc.ca/reports/pdf/perf_rep_2004/index_f.htm

Cornish, D., \& Clarke, R. (1986). The reasoning criminal: Rational choice perspectives on offending. New York: Springer-Verlag.

Côté, G., \& Hodgins, S. (2003). Les troubles mentaux et le comportement criminel. In M. Le Blanc, M. Ouimet \& D. Szabo (2003), Traité de criminologie empirique (503-548). Montréal: Les Presses de l'Université de Montréal.

Cusson, M., \& Pinsonneault, P. (1986). The decision to give up crime. In D. Cornish \& R. Clarke (Eds.), The reasoning criminal: Rational choice perspectives on offending (72-82). New York: Springer-Verlag. 
Debuyst, C. (2010). Essais de criminologie clinique. Entre psychologie et justice pénale. Bruxelles: Éditions Larcier.

De Greeff, E. (1927). Un vagabond délirant méconnu pendant trente années. (Contributions à l'étude de la délinquance des schizophrènes). Revue de droit pénal et de criminologie, 11 (novembre), 1004-1014.

De Greeff, E. (1955). Criminogenèse. Rapport général. In Actes du II congrès international de criminologie (267-306). Paris: Presses Universitaires de France.

De Greeff, E. (1956). Le devenir, élément du processus criminogène; la durée, condition de son étude. In L'bomme criminel. Études d'aujourd'bui (169-192). Louvain: Éditions Nauwelaerts.

Elder, G. H., \& Shanahan, M. J. (2006). The life course and human development. In W. Damon \& R. M. Lerner (Eds.), Handbook of child psychology: Theoretical models of human development (665-716). New York: John Wiley.

Farrall, S. (2004). Social capital and offender reintegration: making probation desistance focussed. In S. Maruna \& R. Immarigeon (Eds.), After Crime and Punishment: Pathways to Offender Reintegration (57-84). Cullompton: Willan.

Farrall, S., \& Calverley, A. (2006). Understanding Desistance from Crime: Theoretical Directions in Resettlement and Rebabilitation Maidenhead. Berkshire: Open University Press.

Gabbard, G. O., \& Williams, P. (2001). Preserving Confidentiality in the Writing of Case Reports. International Journal of Psycho-Analysis, 82, 10671068.

Gadd, D., \& Farrall, S. (2004), Criminal careers, desistance and subjectivity: Interpreting men's narratives of change. Theoretical Criminology, $8(2), 123-$ 156.

Giorgi, A. (1985). Phenomenology and psychological research. Pittsburgh: Duquesne University Press.

Giorgi, A. (1997). The theory, practice, and evaluation of the phenomenological method as a qualitative research procedure. Journal of Phenomenological Psychology, 28 (2), 235-260.

Glueck, S., \& Glueck, E. T. (1940). Later Criminal Careers. New York: Commonwealth Fund.

Gottfredson, M., \& Hirschi, T. (1990). A general theory of crime. Stanford, CA: Stanford University Press.

Gove, W. (1985). The effect of age and gender on deviant behaviour. In A. S. Rossi (Ed.), Gender and The Life Course. New York: Aldine.

Hare, R. D. (1991). The Hare Psychopatby Checklist-Revised. Toronto: Multi-Health Systems.

Harper, S. (2005). Opposition getting tough on crime legislation: Harper. 25 octobre 2006. Disponible sur: http://www.cbc.ca/canada/story/2006/10/25/ harper-bills.html

Hirschi, T. (1969). Causes of delinquency. Berkeley: University of California Press.

Instrup, O. (1992). Rapport du Groupe d'étude sur les longues sentences. Canada: Service correctionnel du Canada. 
Joos, J., \& Debuyst, C. (1968). L'évolution vers la délinquance et le T. A. T. comme moyen de diagnostic. In C. Debuyst (Ed.), La criminologie clinique (274-299). Orientations actuelles. Bruxelles: Charles Dessart.

Kvale, S. (1983). The Qualitative Research Interview. Journal of Phenomenological Psychology, 14, 171-196.

Landreville, P. (2004). Réinsertion dans le marché du travail: L'impact des antécédents judiciaires. In J. Poupart, S. Arcand \& J. Cantin (Eds.), Au-delà du système pénal: l'intégration sociale et professionnelle de groupes judiciarisés et marginalisés (213-230). Montréal: Presses de l'Université du Québec.

Laub, J. H., \& Sampson, R. J. (2001). Understanding desistance from crime. In M. Tonry (Ed.), Crime and justice: A review of research (1-69). Chicago: University of Chicago Press.

Laub, J. H., \& Sampson, R. J. (2003). Shared beginnings, divergent lives: Delinquent boys to age 70. Cambridge, MA: Harvard University Press.

Marchetti, A-M. (2001). Perpétuités: le temps infini des longues peines. Paris: Plon Terre Humaine.

Mailloux, N. (1971). Jeunes sans dialogue. Paris: Fleurus.

Maruna, S. (2001). Making Good. Washington, DC: APA Press.

Maruna, S., \& Immarigeon, R. (2004). After Crime and Punishment: Patbways to Offender Reintegration. Cullompton: Willan.

Maruna, S., \& Roy, K. (2007). Amputation or reconstruction? Notes on the concept of "knifing off" and desistance from crime. Journal of Contemporary Criminal Justice, 23 (1), 104-124.

Motiuk, L., \& Nafekh, L. (1997). Characteristics of Life Sentenced Offenders in Federal Institutions. Canada: Service correctionnel du Canada, Division de la recherche, janvier 1997.

National Parole Board (1999). Repeat Homicide Offences Committed by Offenders Under Community Supervision. Canada: Gouvernement du Canada, Commission nationale des libérations conditionnelles. Disponible sur: www.npbcnlc.gc.ca/reports/pr101001_e.htm

Polkinghorne, D. E. (1989). Phenomenological Research Methods. In R. S. Valle \& S. Halling (Eds.), Existential-phenomenological Perspectives in Psycho$\log y$ : Exploring the Breadth of Human Experience (41-60). New York: Plenum Press.

Poupart, J. (1997). L'entretien de type qualitatif: Considérations épistémologiques, théoriques et méthodologiques. In J. Poupart, J. P. Deslauriers, L. H. Groulx, A. Laperrière, R. Mayer \& A. P. Pires (Eds.), La recherche qualitative: enjeux épistémologiques et méthodologiques (173-209). Boucherville: Éditions Gaëtan Morin.

Sampson, R. J., \& Laub, J. H. (1993). Crime in the making: Patbways and turning points through life. Cambridge, MA: Harvard University Press.

Sampson, R. J., \& Laub, J. H. (2003). Life course desisters? Trajectories of crime among delinquent boys followed to age 70. Criminology, 41, 555-592.

Thériault, Y. (2005). Tout le monde debors. Montréal: Libre Expression/Québécor Media. 
Warr, M. (2002). Companions in crime. Cambridge, UK: Cambridge University Press.

\begin{abstract}
The objective of this article is to explore life trajectories of prisoners serving life sentences that have been granted complete parole and are living in the community, in order to better understand processes of psychological change. A qualitative methodology was chosen in order to establish the life narratives of 20 prisoners serving life sentences but living in the community having been granted parole. A series on some 15 two hour-long interviews of each participant are realized in order to establish their life trajectory. Two dimensions have emerged following analysis of the interview material that have been designated as the pivotal moment and the turning point. The life story of Laurent is presented with a view of illustrating and discussing these results. Finally, a short psychoanalytic discussion of the results is presented as concluding remarks.
\end{abstract}

KEYWORDS - Parole, life sentence, process of change, pivotal moments, turning point, life trajectory, life story.

RESUMEN - El presente artículo explora la trayectoria de vida de detenidos condenados a perpetuidad pero que han beneficiado de una liberación condicional completa, con el fin de comprender mejor el proceso de cambio psicológico que se ha operado en ellos. Para este estudio se eligió emplear una metodología cualitativa con el propósito de trazar una historia de vida de 20 detenidos condenados a cadena perpetua que viven en comunidad. Se prevé una serie de alrededor de 15 entrevistas de dos horas con cada participante para reconstruir su trayectoria de vida. El análisis de los resultados preliminares permite identificar dos dimensiones importantes en dicha trayectoria y de manera específica en el proceso de cambio que experimentaron. Estas dimensiones han sido designadas con las expresiones "momento pivote" (moment charnière) y "punto de cambio" (point tournant). Se presenta la historia de Laurent con el fin de ilustrar estas constataciones y discutir su sentido. Finalmente, como conclusión, se propone una corta discusión psicoanalítica.

PALABRAS CLAVE - Liberación condicional, cadena perpetua, proceso de cambio, momento pivote, punto de cambio. 